\title{
Leishmania infantum-induced primary and challenge infections in rhesus monkeys (Macaca mulatta): a primate model for visceral leishmaniasis
}

\author{
R. Porrozzi ${ }^{a}$, M.S. Pereira ${ }^{a}$, A. Teva ${ }^{a}$, A.C. Volpini ${ }^{a}$, M.A. Pinto ${ }^{b}$, \\ R.S. Marchevsky ${ }^{c}$, A.A. Barbosa Jr ${ }^{d}$, G. Grimaldi Jr ${ }^{a, *}$ \\ a Departamento de Imunologia, FIOCRUZ, Av. Brasil 4365, Rio de Janeiro RJ, 21045-900, Brazil \\ b Departamento de Virologia, Instituto Oswaldo Cruz, FIOCRUZ, Av. Brasil 4365, Rio de Janeiro RJ, 21045-900, Brazil \\ c Laboratório de Neurovirulência, Instituto de Tecnologia em Imunobiológicos, FIOCRUZ, \\ Av. Brasil 4365, Rio de Janeiro RJ, 21045-900, Brazil \\ d Unidade de Histopatologia, Centro de Pesquisas Gonçalo Moniz, FIOCRUZ, Salvador, BA, Brazil
}

Received 1 September 2005; received in revised form 3 November 2005; accepted 3 November 2005 Available online 7 February 2006

\section{KEYWORDS \\ Visceral leishmaniasis; Leishmania infantum; Macaca mulatta; Immune responses; Histopathology; Treatment}

\begin{abstract}
Summary Visceral leishmaniasis (VL) was experimentally induced in rhesus macaques (Macaca mulatta) by intravenously inoculating $2 \times 10^{7}$ amastigotes $/ \mathrm{kg}$ of body weight of Leishmania infantum. The macaques developed a systemic disease showing characteristic features of human VL such as fever, diarrhoea, body weight loss, anaemia, hypergammaglobulinaemia and transient lymphocytosis, as well as lymph node, liver and/or spleen enlargement. Nine weeks after infection, one primate showed pronounced weight loss, became moribund and was euthanized. The necropsy findings included granulomas composed of parasite-containing macrophages, lymphocytes and plasma cells in the liver, spleen and lymph nodes. The remaining macaques had a sustained course of infection but developed a mild-to-moderate illness that subsequently showed evidence of self-cure. Of note, pathological findings included a typical cell-mediated immunity-induced granulomatous reaction that had an effect on the control of parasite replication. All infected monkeys responded with increased production of anti-Leishmania-specific IgG antibodies. Despite the fact that clinical resistance to $L$. infantum was not consistently associated with a parasite-specific cell-mediated immune response, drug-cured macaques from the primary infection acquired immunity to homologous re-infection. These findings point to the feasibility of using the $L$. infantum macaque model for pre-clinical evaluation of novel chemotherapeutics or vaccine candidates for human VL.
\end{abstract}

(c) 2005 Royal Society of Tropical Medicine and Hygiene. Published by Elsevier Ltd. All rights reserved.

\footnotetext{
* Corresponding author. Tel.: +55 213865 8203; fax: +55 2122094110.

E-mail address: grimaldi@ioc.fiocruz.br (G. Grimaldi Jr).
}

0035-9203/\$ - see front matter @ 2005 Royal Society of Tropical Medicine and Hygiene. Published by Elsevier Ltd. All rights reserved. doi:10.1016/j.trstmh.2005.11.005 


\section{Introduction}

Leishmaniasis is one of the major infectious diseases affecting mainly the poorest regions of the world. Among the two million new human infections annually in the 88 countries where the disease is endemic, 500000 cases are clinical visceral leishmaniasis (VL); excluding epidemics, there are 57000 deaths annually worldwide (www.who.int/ $\mathrm{tdr} /$ diseases). Concerns about treatment failure for VL are exacerbated by geographical variation in antimonial treatment regimens, severity of disease and sensitivity of Leishmania species, and no proven successful vaccine for controlling human leishmaniasis is in routine use (Davies et al., 2003).

The protozoa Leishmania donovani and $L$. infantum (syn. L. chagasi) are the causative agents of VL. The incidence is rising with urbanisation and HIV co-infection (Desjeux, 2001). Leishmania infantum VL is a zoonotic disease found in Latin America, Europe, Asia and Africa. In the Neotropics the parasite is usually transmitted by the sandfly Lutzomyia longipalpis, and domestic dogs are the principal reservoir hosts. Control programmes in Brazil focus on residual insecticide spraying and mass elimination of seropositive dogs, but these conventional measures are costly and fail in developing countries (Courtenay et al., 2002).

The artificial induction of protective immunity using second-generation vaccines against leishmaniasis (Coler and Reed, 2005) would be essential for ultimate control of the disease. Nevertheless, there is still much to be done in assessing the effectiveness of vaccination in the absence of a natural challenge. Clinical development of the vaccine comprising the polyprotein Leish-111f in the adjuvant MPL-SE (Coler and Reed, 2005) may not be fully protective across Leishmania species. Another vaccine candidate of specific interest is HASPB1, a recombinant stage-specific hydrophilic acylated surface protein that protects against experimental L. donovani LV9 in mice (Stager et al., 2000).

Non-human primates are physiologically close to humans (Kennedy et al., 1997) and will probably mimic human response to $L$. donovani complex parasites. Indeed, different Old World monkeys and Neotropical simian species have become useful in studying the biology of infection and in dissecting the host response to these parasites. Those reported as being highly susceptible to this group of pathogens include New World primates such as the owl monkey Aotus trivirgatus (Broderson et al., 1986; Chapman et al., 1981), the squirrel monkey Saimiri sciureus (Chapman and Hanson, 1981; Dennis et al., 1985, 1986) and the marmoset Callithrix jacchus jacchus (Marsden et al., 1981). All of these species have since been used as non-human primate models of VL for antileishmanial chemotherapy studies (Berman et al., 1986; Madindou et al., 1985). East African primate species such as Sykes monkeys (Cercopithecus mitis) and baboons (Papio cynocephalus) all supported low-grade $L$. donovani infections for periods ranging between 4 and 8 months and subsequently showed evidence of self-cure (Githure et al., 1986). Furthermore, disease mimicking human VL was established in Indian langur monkeys Presbytis entellus (Dube et al., 1999) as well as in vervet monkeys Cercopithecus aethiops (Binhazim et al., 1993; Gicheru et al., 1995). The $L$. donovani-langur monkey model was also explored to assess different vaccine formulations against VL (Dube et al., 1998; Misra et al., 2001).

In our studies, L. amazonensis (Amaral et al., 1996), L. major (Amaral et al., 2001) and L. braziliensis (Teva et al., 2003) infections in outbred rhesus macaques produced cutaneous leishmaniasis (CL) of variable severity (ranging from self-healing granulomatous cutaneous lesions to non-healing mucocutaneous disease) and the immunological findings were similar to those observed in human CL. The homology between the Macaca mulatta immune system (Giavedoni, 2005; Pahar et al., 2003) and that of humans has led to the belief that the efficacy of those antigens selected as being candidates for an anti-Leishmania vaccine in this animal model (Campos-Neto et al., 2001; Kenney et al., 1999) may represent suitable vaccine candidates against this parasite in humans. The present studies on infections with $L$. infantum in macaques were performed to develop an animal model for human VL that would optimise progression of vaccine candidates to phase I trials. The parallels found between humans and this primate species with regard to susceptibility, clinicopathological changes and specific immunological responses to $L$. infantum visceral infections are not surprising given their close phylogenetic relationship (Kennedy et al., 1997), thus providing an acceptable model for the purpose of vaccine evaluation.

\section{Material and methods}

\subsection{Animals}

Colony outbred (Andrade et al., 2004a), young adult (weighing $6-10 \mathrm{~kg}$ ) male rhesus macaques (M. mulatta) from the Fiocruz Primate Research Centre (Rio de Janeiro, Brazil) were used in this study. Primates were housed individually in stainless-steel squeeze-back cages and fed daily with a commercially available primate diet supplemented with fresh fruits and vegetables. Water was provided ad libitum. Syrian golden hamsters (Mesocricetus auratus) were also used in this study as the donor source of the inoculums. The experimental protocols involving monkeys and hamsters were reviewed and approved by the Institutional Animal Care and Use Committee (CEUAFiocruz, resolution \# P0048-00). Primates were acclimatised to the laboratory conditions for at least 2 weeks before the experimental procedures began; they were monitored daily by non-human primate care specialists and evaluated by a veterinarian. When necessary (before infection and prior to each sampling or testing procedure), the animals were anaesthetised with $10-20 \mathrm{mg}$ ketamine hydrochloride $\left(\operatorname{Vetalar}^{\top M}\right) / \mathrm{kg}$ of body weight injected intramuscularly.

\subsection{Experimental infections}

The strain (MHOM/BR/2000/1669) of L. infantum originally isolated from the bone marrow of a Brazilian patient with active VL was maintained by hamster-to-hamster passage. The parasite was typed by multilocus enzyme electrophoresis (Cupolillo et al., 1994) in our laboratory before being used for infection. For experimental infections, amastigotes harvested from heavily infected hamster spleens were prepared 
according to the procedure as previously described (Amaral et al., 1996).

A study was first designed to provide information regarding the virulence of the infecting parasite. Six of the seven naïve macaques were each inoculated via the saphenous vein with $2 \times 10^{7}$ amastigotes $/ \mathrm{kg}$ of body weight. One monkey, designated as the control, was injected with uninfected hamster spleen homogenate. An additional study was designed to evaluate the level of clinical resistance to the homologous challenge. These same macaques that had recovered from $V L$ following treatment with meglumine antimoniate (at a dosage of $20 \mathrm{mg}$ of $\mathrm{Sb}^{v} / \mathrm{kg} /$ day, given intramuscularly for 28 days; therapy start at week 37 after infection) were each subsequently challenged via the saphenous vein with $2 \times 10^{8}$ amastigotes/ $\mathrm{kg}$ at week 60 post inoculation (PI).

\subsection{Assessment of infection and disease development}

Once every 2 weeks, all macaques underwent gross physical examination and body weight, blood samples and rectal temperature were taken from each monkey through the duration of the experiment. Monkeys were examined for six typical signs of human VL (fever, diarrhoea, body weight loss, lymphadenopathy, hepatomegaly and splenomegaly). Following the procedure described by Courtenay et al. (2002), each sign was scored on a semiquantitative scale from 0 (absent) to 3 (severe) and these scores were added together to give an overall clinical score. Monkeys with a total score of 0-2 were arbitrarily classed as asymptomatic, those with a score of 3-6 were classified as oligosymptomatic and those with a score of 7-18 were classified as polysymptomatic.

Heparin- or EDTA-anticoagulated blood samples were collected by venipuncture for haematological and immunological studies. Blood collected into tubes containing EDTA as an anticoagulant was used for a complete haemogram. The following blood components were measured with a computer-directed analyser, using commercially available kits (CELM ${ }^{\top M}$ Cia Equipadora de Laboratórios Modernos, Barueri, SP, Brazil): cholesterol, urea nitrogen, total protein and albumin, alanine aminotransferase (ALT) and aspartate aminotransferase (AST). Total erythrocyte, leukocyte and haemoglobin counts were carried out with the cellular counter 530/550 (CELM ${ }^{T M}$ Cia Equipadora de Laboratórios Modernos). Haematocrit values were performed according to standard procedures, including Wright's coloration, which was used for blood films. Commercial assays were conducted in accordance with the manufacturer's instructions. Total plasma proteins were determined with a refract meter (American Optical T/C, Scientific Instrument Division, Buffalo, NY, USA). The plasma protein patterns of each monkey were determined by cellulose acetate electrophoresis using a Modular Electrophoresis System (Roche Laboratories, Basel, Switzerland).

For assessment of parasites, biopsy specimens were removed from bone marrow, liver and spleen at distinct stages of infection and processed for DNA isolation and culture or histological examination. For PCR analysis, liver and spleen specimens from the uninfected monkey were included as negative controls. Biopsy samples were cultured using NNN blood agar medium overlaid with Schneider's Drosophila insect medium (Sigma, St Louis, MO, USA) supplemented with $20 \%(\mathrm{v} / \mathrm{v})$ heat-inactivated fetal calf serum (HI-FCS) and $100 \mu \mathrm{g} / \mathrm{ml}$ gentamicin (complete Schneider's medium). One macaque that developed very severe disease was euthanized with sodium pentobarbitone (Euthano ${ }^{\circledR}$ ). Gross and light microscopic examinations of the major organs were performed at necropsy. Paraffin sections from biopsy and necropsy tissues samples (fixed in 10\% neutral buffered formalin) were stained with haematoxylin-eosin.

\subsection{Diagnosis by DNA analysis}

The PCR methods followed for detection of Leishmania DNA in infected monkeys have been described previously (Volpini et al., 2004). Briefly, DNA purification from $10-20 \mathrm{mg}$ biopsy fragments of liver, spleen or bone marrow was done using the Wizard ${ }^{\circledR}$ Genomic DNA Purification System (Promega, Madison, WI, USA) following the manufacturer's instructions. Primers were designed to amplify the conserved region of the minicircle of Leishmania kDNA producing genus-specific fragments of $120 \mathrm{bp}$ (Degrave et al., 1994). The reaction was carried out using $1 \mu \mathrm{M}$ of each primer (150: 5' GGG(G/T)AGGGGCGTTCT(C/G)CGAA 3'; and 152: 5' (C/G)(C/G)(C/G)(A/T)CTAT(A/T)TTACACCAACCCC 3') together with $200 \mu \mathrm{M}$ of dNTPs, $0.8 \mathrm{U}$ of Taq DNA polymerase (Invitrogen, São Paulo, Brazil), buffer $(10 \mathrm{mM}$ Tris- $\mathrm{HCl} \mathrm{pH}$ $8.6,50 \mathrm{mM} \mathrm{KCl}, 1.5 \mathrm{mM} \mathrm{MgCl}_{2}$ ) and $2 \mu$ l of DNA template in a final volume of $25 \mu \mathrm{l}$. Amplification was carried out in an MJ Research PTC-200 machine (MJ Research Inc., Watertown, MA, USA) using an initial denaturing step at $95^{\circ} \mathrm{C}$ for $5 \mathrm{~min}$, followed by 29 cycles at $95^{\circ} \mathrm{C}$ for $1 \mathrm{~min}, 55^{\circ} \mathrm{C}$ for $30 \mathrm{~s}, 72^{\circ} \mathrm{C}$ for $10 \mathrm{~s}$ and a final extension step of $5 \mathrm{~min}$. Each experiment included a positive control (100 fg of Leishmania kDNA) and a negative control with no DNA. The expected amplification products of $120 \mathrm{bp}$ were analysed by $8 \%$ polyacrylamide gel in a Mini-Protean II apparatus (Bio-Rad, Hercules, CA, USA) and stained using a DNA Silver Staining Kit (Amersham Biosciences, Buckinghamshire, UK) to visualise the bands. All PCR-negative results were tested for reaction inhibitors by adding $100 \mathrm{pg}$ of total $L$. infantum DNA in a new amplification procedure, which then yielded a positive signal.

\subsection{Antigens}

Promastigotes of L. infantum (MHOM/BR/2000/1669) provided the source of the antigens. A preparation of soluble leishmanial antigens (SLA) was made as described previously (Amaral et al., 2001). The SLAs were used at different concentrations for ELISA and in vitro blast transformation assays.

\subsection{ELISA for antiparasitic antibodies}

Serum from the monkeys was analysed by adapting a standard ELISA technique (Amaral et al., 2001) to detect parasite-specific antibodies (using a peroxidase conjugate rabbit anti-monkey immunoglobulin G; Sigma, St Louis, MO, USA). The reaction was revealed with biotin-avidin peroxidase system. The substrate consisted of $0.04 \%$ 
o-phenylenediamine dihydrochloride and $0.012 \%$ hydrogen peroxidase in phosphate-citrate buffer, $\mathrm{pH} 5$. A group of sera with previously known titres as control values, as well as naïve rhesus controls, were included in each test.

\subsection{Assessment of delayed-type hypersensitivity (DTH) responses}

Animals were assessed for DTH reactions according to the method used for humans (leishmanin skin test (LST)). The antigen (leishmanin), provided by the Fiocruz, Biomanguinhos Unit (Rio de Janeiro, Brazil), consisted of pooled heatkilled cross-species promastigotes suspended in phosphatebuffered saline (PBS) with $0.5 \%$ phenol. For the LST, a volume of $0.1 \mathrm{ml}$ containing macromolecules from $5 \times 10^{6}$ parasites was inoculated intradermally into the forearm and the induration produced was measured $72 \mathrm{~h}$ later. Induration diameters of $\geq 5 \mathrm{~mm}$ were considered positive.

\subsection{Assessment of the in vitro blastogenic and cytokine responses}

The antigen-specific recall proliferative and cytokine responses were measured in peripheral blood leukocytes (PBL) using phytohaemagglutinin (PHA-P) (Sigma, St Louis, $\mathrm{MO}$, USA) stimulation as a positive control. Basic experimental protocols have been reported elsewhere (Amaral et al., 1996). Briefly, purified PBL concentration was adjusted to $5 \times 10^{6}$ cells $/ \mathrm{ml}$ in RPMI-1640 supplemented with $20 \%$ HI-FCS, $2 \mathrm{mM}$ L-glutamine, $100 \mathrm{IU} / \mathrm{ml}$ penicillin, $100 \mu \mathrm{g} / \mathrm{ml}$ streptomycin and $5 \times 10^{-5} \mathrm{M} \beta$-mercaptoethanol (Merck, Darmstadt, Germany). Aliquots $(0.1 \mathrm{ml})$ of the cell suspension were distributed in triplicate cultures in 96-well roundbottomed microtitre plates (Nunc, Roskilde, Denmark). PHA$P$ and SLA were added in $0.1 \mathrm{ml}$ of the same media to a final concentration of $12.5 \mu \mathrm{g} / \mathrm{ml}$ and $10 \mu \mathrm{g} /$ well, respectively. Cultures were incubated at $37{ }^{\circ} \mathrm{C}$ in a humidified $5 \%$ $\mathrm{CO}_{2}$ atmosphere for 3 days in the case of PHA-P or for 4 days in the case of SLA. The cells were pulsed with $1 \mu \mathrm{Ci}$ of ${ }^{3} \mathrm{H}$-thymidine (specific activity $74 \mathrm{GBq} / \mathrm{nmol}$; Amersham International, Buckinghamshire, UK) for the last $24 \mathrm{~h}$ before cell harvest. Radioactivity uptake was measured by liquid scintillation counting. The stimulation index (SI) was determined as the ratio of the mean counts per minute (cpm) of cultures stimulated with PHA-P or SLA to the mean cpm of cultures with RPMI alone (control cultures). A SI $\geq 3$ was considered significant based on the 95th percentile of preinfection values of seven macaques.

Concentrations of IFN- $\gamma$ and IL- 5 in culture supernatants of stimulated cells were measured by ELISA. Cell-free culture supernatants were harvested by centrifugation at $200 \times g$ for $10 \mathrm{~min}$ and cytokine levels were estimated using either a rhesus monkey IFN- $\gamma$ (Biosource, Camarillo, CA, USA) or human IL-5 (R\&D Systems Inc., Minneapolis, MN, USA) commercial ELISA kit. The procedure was carried out following the manufacturer's instructions. In brief, plates were coated with an optimum concentration $(2 \mu \mathrm{g} / \mathrm{ml})$ of mouse anti-rhesus IFN- $\gamma$ or anti-human IL- 5 monoclonal antibody (mAb). Then, $100 \mu$ l of cell culture supernatants and multiple dilutions of the standard $(0-1400 \mathrm{pg} / \mathrm{ml})$ were added in duplicate. Detection was performed by the addition of horseradish peroxidase-streptavidin conjugate at a concentration of 1:2000 in PBS. Reaction was developed using tetramethylbenzidine as the substrate. Cytokine concentrations for unknown samples and controls were read from the plotted standard curve.

\subsection{Statistical analysis}

Student's $t$-test was used in comparative analysis and a $P$ value of $<0.05$ was considered statistically significant. Concordance between cellular immune responses was assessed as reported elsewhere (Amaral et al., 2001).

\section{Results}

\subsection{Establishment of infection and disease development}

Leishmania infantum primary infection in outbred macaques was assessed through time by parasitological examination or PCR of the liver, spleen and bone marrow. All of the monkeys had established infection by week $2 \mathrm{PI}$ and parasites persisted in lymphoid organs as long as 25 weeks $\mathrm{PI}$, except for the monkey (045) that recovered spontaneously from VL (Table 1). Overall, attempts to detect parasite-positive specimens by culture $(61 \%$; 14/23) were more successful than PCR $(53 \% ; 17 / 32)$ and direct microscopic examination $(50 \% ; 9 / 18)$. However, there was variation in the sensitivity of each diagnostic test following the time course of infection. Considering only macaques positive for Leishmania DNA by PCR, the sensitivity of the test was high $(90.9 \%$; $10 / 11)$ during active infection, but lower $(20 \%$; $2 / 10$ samples) in the self-healing phase and after antimony therapy.

Table 2 shows that a similar picture to human VL, characterised by systemic disease of varying severity, develops in L. infantum-infected monkeys. According to their clinical condition, two macaques (N13 and 059) with a total score of 10 and 7 , respectively, and four others $(045,021, \mathrm{~F} 14,043)$ with scores of 4-6 were classed as polysymptomatic and oligosymptomatic, respectively. The most consistent clinical parameters were an intermittent rise in body temperature by $2-4^{\circ} \mathrm{C}$, diarrhoea and body weight loss. These changes were evident by week $2 \mathrm{PI}$ and became more pronounced during the first 4 months of infection. Although visceral organ enlargement was seen as a result of the experimental infection, no animal showed a syndrome similar to human $\mathrm{VL}$ with progressive hepatosplenomegaly.

Nine weeks after infection, one primate (N13) developed a severe illness after losing $42 \%$ of his body weight. At this time he was anorectic, listless and became weak and dehydrated, and was euthanized. Gross necropsy findings included enlarged spleen (five-fold), mesenteric lymph nodes and fatty liver. The surviving five chronically infected macaques lost between $7 \%$ and $28 \%$ of their body weight. These primates clinically recovered from infection following antimony-based therapy and regained normal weight by week $45 \mathrm{PI}$. At this time, the weight of the uninfected control monkey $(015)$ increased by $5 \%$. Of note, in the second experiment we found that the same macaques were refractory to the disease (as indicated by complete clinical resistance) 
Table 1 Comparative results of conventional diagnostic procedures and PCR of Leishmania infantum visceral infection in rhesus macaques (Macaca mulatta)

\begin{tabular}{|c|c|c|c|c|c|}
\hline \multirow[t]{2}{*}{ Monkey } & \multirow[t]{2}{*}{ Time PI (weeks) } & \multirow[t]{2}{*}{ Clinical status (stage) } & \multicolumn{3}{|c|}{ Demonstration of the parasite (biopsy specimen) } \\
\hline & & & Histological sections $^{a}$ & In vitro culture & $P C R^{b}$ \\
\hline 015 & 0 & Uninfected control & n.d. & n.d. & $-(L) ;-(S)$ \\
\hline N13 & $\begin{array}{l}2 \\
5 \\
9\end{array}$ & $\begin{array}{l}\text { Oligosymptomatic (active) } \\
\text { Polysymptomatic (active) } \\
\text { Polysymptomatic (active) }\end{array}$ & $\begin{array}{l}+(L) \\
+(L) \\
+(L)\end{array}$ & $\begin{array}{l}+(S) \\
\text { n.d. } \\
+(S)\end{array}$ & $\begin{array}{l}+(\mathrm{L}) \\
+(\mathrm{BM}) ;+(\mathrm{S}) \\
+(\mathrm{L}) ;+(\mathrm{S})\end{array}$ \\
\hline 059 & $\begin{array}{l}2 \\
5 \\
25 \\
45\end{array}$ & $\begin{array}{l}\text { Oligosymptomatic (active) } \\
\text { Polysymptomatic (active) } \\
\text { Oligosymptomatic (healing) } \\
\text { Asymptomatic (drug-cured) }\end{array}$ & $\begin{array}{l}+(L) \\
+(L) \\
-(B M) ;-(L) \\
\text { n.d. }\end{array}$ & $\begin{array}{l}+(\mathrm{L}) \\
+(\mathrm{BM}) ;+(\mathrm{L}) \\
-(\mathrm{L}) \\
-(\mathrm{L})\end{array}$ & $\begin{array}{l}+(\mathrm{S}) \\
+(\mathrm{BM}) ;+(\mathrm{S}) \\
+(\mathrm{BM}) ;+(\mathrm{L}) \\
-(\mathrm{L})\end{array}$ \\
\hline F14 & $\begin{array}{l}2 \\
5 \\
25 \\
45\end{array}$ & $\begin{array}{l}\text { Oligosymptomatic (active) } \\
\text { Oligosymptomatic (active) } \\
\text { Oligosymptomatic (healing) } \\
\text { Asymptomatic (drug-cured) }\end{array}$ & $\begin{array}{l}+(L) \\
-(L) \\
-(L) \\
\text { n.d. }\end{array}$ & $\begin{array}{l}+(L) \\
-(L) \\
+(B M) \\
-(L)\end{array}$ & $\begin{array}{l}+(\mathrm{S}) \\
-(\mathrm{BM}) ;-(\mathrm{S}) \\
-(\mathrm{BM}) ;-(\mathrm{S}) \\
-(\mathrm{L})\end{array}$ \\
\hline 043 & $\begin{array}{l}2 \\
5 \\
25 \\
45\end{array}$ & $\begin{array}{l}\text { Oligosymptomatic (active) } \\
\text { Oligosymptomatic (active) } \\
\text { Oligosymptomatic (healing) } \\
\text { Asymptomatic (drug-cured) }\end{array}$ & $\begin{array}{l}+(L) \\
-(L) \\
-(L) \\
\text { n.d. }\end{array}$ & $\begin{array}{l}+(\mathrm{L}) \\
+(\mathrm{BM}) \\
+(\mathrm{L}) \\
-(\mathrm{L})\end{array}$ & $\begin{array}{l}\text { n.d. } \\
\text { n.d. } \\
-(B M) ;-(S) \\
-(L)\end{array}$ \\
\hline 021 & $\begin{array}{l}2 \\
5 \\
25 \\
45\end{array}$ & $\begin{array}{l}\text { Oligosymptomatic (active) } \\
\text { Oligosymptomatic (active) } \\
\text { Oligosymptomatic (active) } \\
\text { Asymptomatic (drug-cured) }\end{array}$ & $\begin{array}{l}+(L) \\
\text { n.d. } \\
-(L) \\
\text { n.d. }\end{array}$ & $\begin{array}{l}+(\mathrm{L}) \\
-(\mathrm{BM}) \\
+(\mathrm{BM}) \\
-(\mathrm{L})\end{array}$ & $\begin{array}{l}+(\mathrm{S}) \\
+(\mathrm{L}) ;+(\mathrm{S}) \\
+(\mathrm{BM}) ;-(\mathrm{S}) \\
-(\mathrm{L})\end{array}$ \\
\hline 045 & $\begin{array}{l}2 \\
5 \\
25 \\
45\end{array}$ & $\begin{array}{l}\text { Oligosymptomatic (active) } \\
\text { Oligosymptomatic (active) } \\
\text { Asymptomatic (healing) } \\
\text { Asymptomatic (drug-cured) }\end{array}$ & $\begin{array}{l}+(L) \\
-(L) \\
-(L) \\
\text { n.d. }\end{array}$ & $\begin{array}{l}+(L) \\
\text { n.d. } \\
-(B M) \\
-(L)\end{array}$ & $\begin{array}{l}+(\mathrm{S}) \\
+(\mathrm{BM}) ;-(\mathrm{S}) \\
-(\mathrm{BM}) ;-(\mathrm{S}) \\
-(\mathrm{L})\end{array}$ \\
\hline
\end{tabular}

PI: post inoculation; BM: bone marrow; L: liver; S: spleen; +: positive; -: negative; n.d.: not done.

a Haematoxylin-eosin stain.

b Each experiment included positive and negative controls (see Section 2.4).

when re-challenged by homologous leishmanial parasites at 60 weeks after the primary infection.

\subsection{Haematology and blood chemistry}

The results for the haematological parameters evaluated in the rhesus macaques prior to and at different time points after $L$. infantum challenge infections are given in Table 3. The pre-infection mean blood counts were within the normal range compared with the baseline haematological data previously found for clinically healthy rhesus macaques from the Fiocruz colony (Andrade et al., 2004b). The most consistent haematological finding was a normchromic, normocytic anaemia. An appreciable fall in the red blood cell (RBC) count occurred from week 2 PI onward, and the count was minimal at 46 weeks $\mathrm{PI}$. Packed cell volumes and haemoglobin levels paralleled the RBC values, but exhibited a wide variation. White blood cell counts also varied during infection. Whilst the haemogram revealed neutrophilia and lymphopenia by 9 weeks PI, neutropenia, eosinophilia and lymphocytosis were detected at week $15 \mathrm{PI}$. Alterations in serum chemistries of infected macaques included increases in blood urea nitrogen, total protein and globulin. Of note, in at least three of these macaques the plasma concentration of gamma globulins increased from a pre-inoculation mean value of $0.3 \mathrm{~g} / \mathrm{dl}$ to $0.8 \mathrm{~g} / \mathrm{dl}$ at week $9 \mathrm{PI}$ (Table 2). Typical changes are also illustrated in Figure 1.

One monkey (N13) that had severe disease showed significantly altered haematological values. The RBC values decreased from a pre-infection value of $5.695 \times 10^{6} / \mathrm{ml}$ to $3.300 \times 10^{6} / \mathrm{ml}$ by 6 weeks PI. Packed cell volumes and haemoglobin levels decreased from $38 \%$ and $13.3 \mathrm{~g} / \mathrm{dl}$ at pre-infection to $20 \%$ and $7.4 \mathrm{~g} / \mathrm{dl}$, respectively, at 6 weeks $\mathrm{PI}$. The leukocyte count reduced from an initial value of $6.20 \times 10^{3} / \mathrm{ml}$ to $1.90 \times 10^{3} / \mathrm{ml}$. At this time, the levels of AST $(72 \mathrm{IU} / \mathrm{l})$ or ALT $(88 \mathrm{IU} / \mathrm{l})$ were elevated, but the cholesterol level declined $(108 \mathrm{mg} / \mathrm{dl})$ in this animal.

\subsection{Histopathological findings}

Hepatic granulomas were found in all infected macaques. Granulomas were formed as early as 2 weeks PI and persisted as active in some monkeys through at least 33 weeks PI. The poorly differentiated granulomas of the initial infection consisted primarily of widespread macrophages (some of which contained amastigotes) and variable numbers of 
Table 2 Clinical outcome of experimental Leishmania infantum visceral leishmaniasis in rhesus macaques (Macaca mulatta)






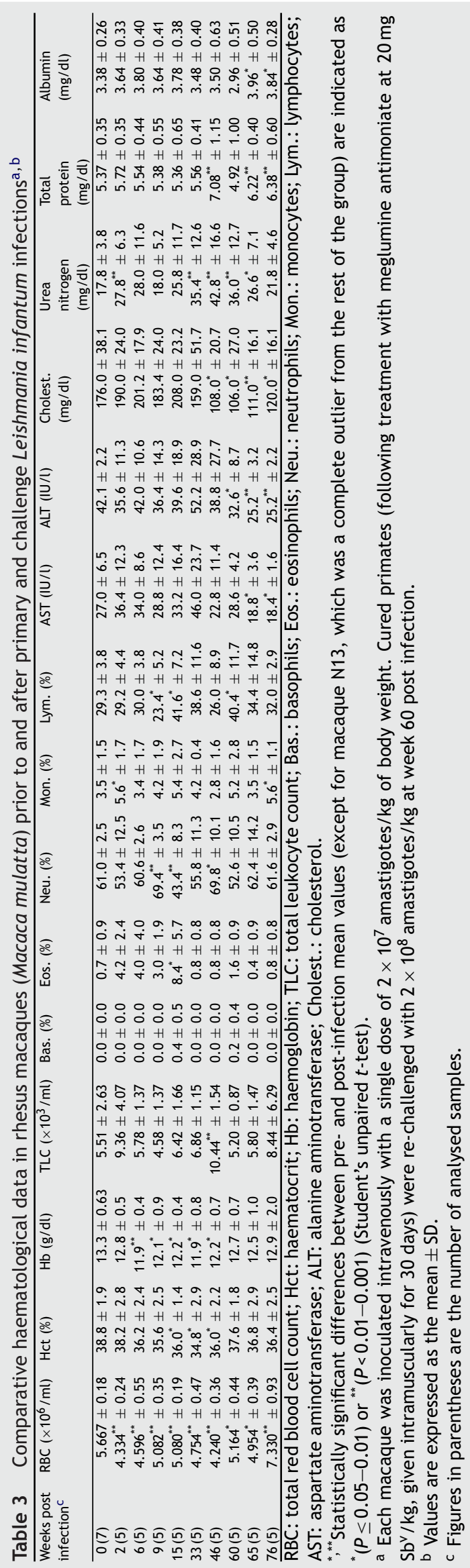

lymphocytes, plasma cells and occasional neutrophils. This inflammatory process evolved to form well circumscribed, well differentiated granulomas, with epithelioid cells fusing to form Langhans-type multinucleated giant cells, surrounded by lymphocytes and occasionally plasma cells (Figure $2 \mathrm{~A}$ and $\mathrm{B}$ ). These granulomas contained less marked parasitised macrophages at week $9 \mathrm{PI}$ compared with those of the initial infection. Additional histological findings in the liver included proliferation and hyperplasia of parasiteladen Kupffer cells, associated with diffuse fatty changes in the stellate cells (Figure $2 \mathrm{C}$ and D). Amastigote-containing macrophages were also seen in the spleen, lymph nodes and bone marrow (data not shown). Sections from the spleen revealed that lymphoid follicles increased in number and size, as well as congested red pulp, with reticular cells proliferating and macrophages (some of which contained haemosiderin) aggregating in the cords and walls of dilated sinuses (Figure 2E and F). Following therapy, older hepatic granulomas observed in portal zones by week $45 \mathrm{PI}$ (the latest time that biopsy samples were taken) developed an enclosing rim of fibroblasts and connective tissue (data not shown). In addition, parasites were not found in sections at this time.

\subsection{Humoral responses}

As shown in Figure 3, the antibody responses detected by ELISA in experimental macaques varied following infection. The serum levels of $L$. infantum-specific IgG antibodies reached a plateau by 15 weeks $\mathrm{PI}$ and subsequently declined slightly after antimonial therapy. Following re-challenge, these animals had heightened levels of antigen-specific antibodies, continuing up to 76 weeks PI. Interestingly, the antibody titres produced by polysymptomatic macaques (N13 and 059) were not consistently higher compared with oligosymptomatic animals.

\subsection{Cell-mediated immune responses}

A very high degree of variability was observed in the parasite-specific cell-mediated immune responses between individuals over time following infection with $L$. infantum. Marked DTH responses were demonstrated in four macaques by 21 weeks PI (Table 2 ). In contrast, the remaining infected (N13 and 021) and uninfected (015) monkeys injected with the same leishmanial antigen developed either minimal skin indurations (mean score, $<3 \mathrm{~mm}$ ) or no reaction.

Mitogen (PHA)-induced proliferation of PBLs from primates was comparable prior to and throughout infection (data not shown). The parasite-specific recall proliferative and cytokine responses of T-lymphocytes from individual macaques through the course of the experiment are shown in Figure 4. Whereas the specific responses were negative in primates before the challenge, four animals (059, F14, 043 and 045) converted to a positive proliferative response (maximum SI values ranging from 12 to 27 ) after infection (Figure 4A). Of note, one oligosymptomatic monkey (021) demonstrated a specific proliferative response (SI value of 27.5) only by week $76 \mathrm{PI}$. 

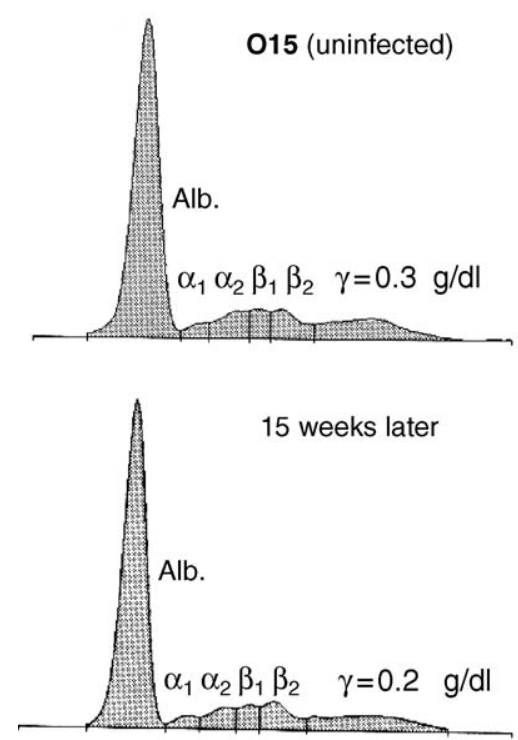

(A)
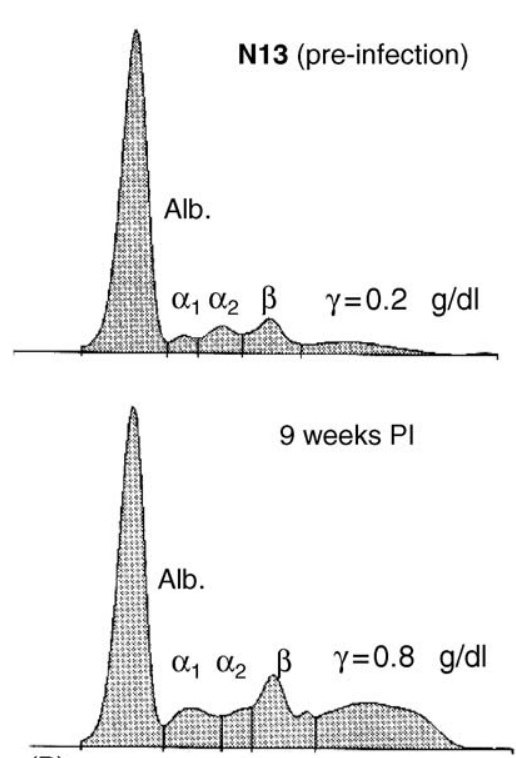

(B)

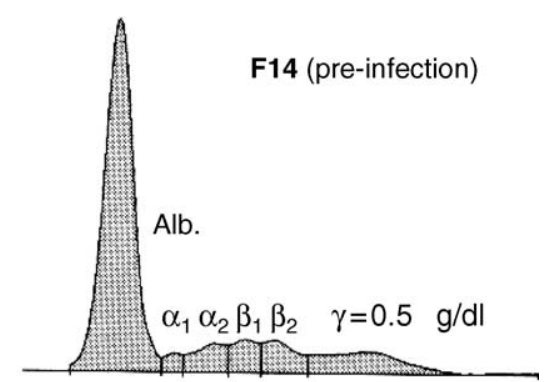

15 weeks $P$

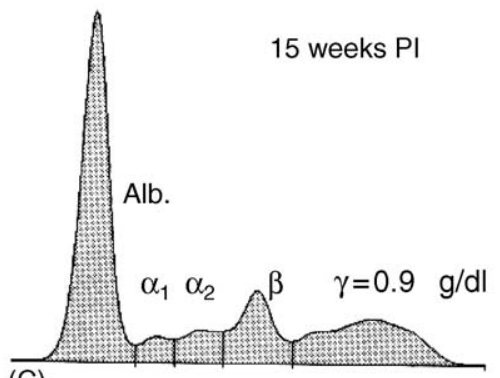

(C)

Figure 1 Typical changes in cellulose acetate electrophoretic patterns of plasma components in two representative Leishmania infantum-infected macaques that developed either acute (N13) or chronic (F14) disease, compared with data obtained from the control monkey (015). Similar changes were observed in other infected macaque (see Table 2). PI: post inoculation.

Significant but varying levels of IFN- $\gamma$ were secreted in PBL cultures from all the infected macaques when stimulated with either PHA (data not shown) or SLA (Table 2; Figure 4B). In the uninfected control monkey (015), IFN$\gamma$ was only detected when PBLs were stimulated with PHA. Noteworthy, a strong Leishmania-specific IFN- $\gamma$ response was elicited in the rhesus monkey (N13) that had the most protracted fatal course of infection. In contrast, either PHA(data not shown) or SLA-induced IL-5 secretion in supernatant fluids was at low or undetectable levels (Table 2; Figure $4 \mathrm{C})$. Comparison of immune responses in terms of antigen-specific IFN- $\gamma$ production with the blastogenic response of PBLs from infected macaques showed that there was no positive correlation between the results of the two assays $\left(r^{2}=0.038 ; P=0.1453\right)$.

\section{Discussion}

Typically, L. infantum VL affects young children and immunocompromised adults. The disease can have a long incubation period and has been reported to present as a smouldering subclinical illness characterised by weight loss, fever and diarrhoea that can evolve either into a self-healing or full-blown disease (Evans et al., 1995). The clinical features of VL in humans include intermittent fever, progressive emaciation, anaemia, lymphadenopathy and hepatosplenomegaly. Although malnutrition is a risk factor for the development of human VL, our understanding of the pathogenesis of the disease is still rudimentary.

Non-human primates have varying degrees of susceptibility to $L$. donovani complex parasites. The results of this study revalidate original reports (Manson-Bahr and Wilson, 1976; Shortt, 1923) identifying M. mulatta as a moderately susceptible host to $L$. donovani complex parasites. In the original model description (Shortt, 1923), induced fulminat- ing infections were regarded as exceptions rather than the rule. Likely, macaques in this study had a sustained course of $L$. infantum visceral infection, ranging from mild selfcuring to severe disease. The macaques lost between $7 \%$ and $42 \%$ of their body weight and developed progressive liver lesions as revealed by the granulomatous inflammatory reaction studied chronologically after infection. Hepatomegaly and splenomegaly were difficult to evaluate in monkeys surviving the disease, but the post-mortem findings detected in rhesus N13 included a five-fold enlarged spleen.

The major haematological changes (anaemia associated with haemosiderosis and tissue iron deposition) in rhesus macaques with $\mathrm{VL}$ paralleled the haemolysis associated with hypersplenism observed to occur in humans with VL (Cartwright et al., 1948) and other infected monkey species (Binhazim et al., 1993; Broderson et al., 1986; Dennis et al., 1985). Moreover, one monkey (N13) that had severe disease showed a significant increase in the levels of AST and ALT, indicating liver disorders. The hepatic lesions resolved following drug-induced recovery from the disease, confirming the antileishmanial efficacy of the reference drug $N$ methylglucamine antimoniate (Glucantime ${ }^{\circledR}$ ) in the rhesus monkey model (Teva et al., 2005).

Sensitive molecular techniques have allowed the identification of leishmanial parasites directly in scars after clinical cure of L. (L.) braziliensis CL in patients (Schubach et al., 1998) and in experimentally infected macaques (Teva et al., 2003). By contrast, whereas persistence of $L$. infantum parasites was documented in all monkeys during active infection, no parasites were found (using either conventional diagnostic procedures or PCR for detecting Leishmania DNA) after treatment. Whether exposure to a particular parasite has led to sterile cure could not be ascertained by this study. The ability of Leishmania to establish latency in immune individuals is evidence of parasite determinants to escape the host's immune response by clonal 

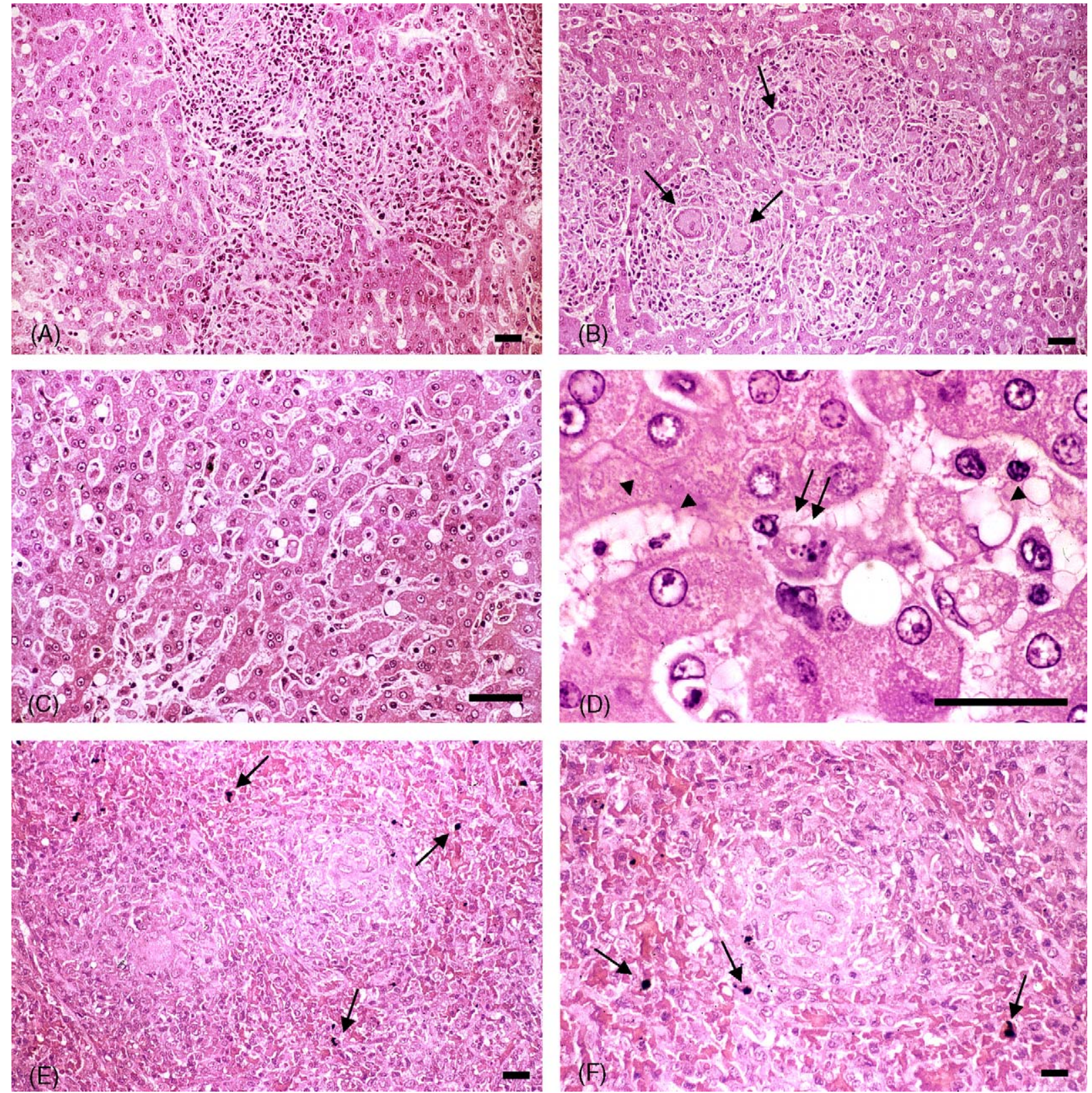

Figure 2 Photomicrographs of the liver and spleen from a rhesus macaque (N13) 9 weeks after inoculation with Leishmania infantum. (A,B) Sections showing multifocal coalescing hepatic immune granulomas consisting of an aggregation of activated macrophages (epithelioid cells) and multiple Langhans-type giant cells (arrows), surrounded by lymphocytes, at the portal spaces, which obliterate the sinusoids and protrude the parenchyma. (C,D) Also illustrated are the proliferation and hyperplasia of parasite-laden Kupffer cells (double arrow), associated with fatty changes of the stellate cells (arrowheads). (E,F) Sections from the spleen showing granulomas consisting of differentiated macrophages in the pulp, with widespread macrophages aggregating in the cords and walls of dilated medullary sinuses. Numerous haemosiderin deposits are present (arrows). (H\&E stain; bar scale =10 $\mu \mathrm{m}$.)

selection or molecular changes resulting from mutational or recombinational processes. Of note, our genotyping analyses showing genetic variations among $L$. (L.) braziliensis isolates from humans and experimentally infected macaques (Teva et al., 2003) indicated that persistent parasites do not retain the characteristics of the parental clones.

It should be noted that our model is artificial in the sense that intravenous injection of a large number of amastigotes initiates the infection, which may account for the parasitic invasion of lymphopoietic organs with the result- ing chronic granulomatous inflammatory response that was observed. Descriptions of the histopathological lesions in the liver either of patients with VL (Sen Gupta et al., 1956) or of experimentally infected $L$. donovani macaques (MansonBahr and Wilson, 1976) resemble the granulomas described here. The hepatic granulomas in this study were most prominent 2-9 weeks PI and had an effect on the control of parasite replication. Of note, the marked regression of immune granulomas and the eventual absence of parasites 25 weeks $\mathrm{PI}$ correlated with satisfactory resolution of the disease. 


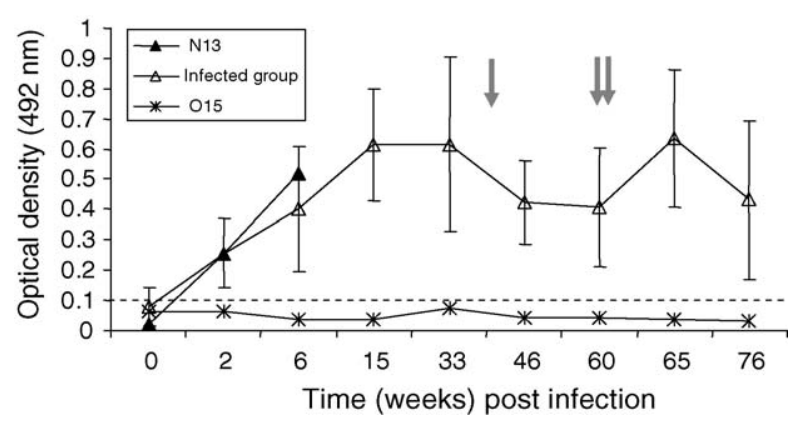

Figure 3 Evolution of serum levels of Leishmania-specific antibodies (lgG) in rhesus macaques following infection with L. infantum. Experimental animals were infected as indicated (Table 2). Following drug-induced recovery from the primary infection (arrow indicates that start of antimonial therapy at week 37 post infection $(\mathrm{PI})$ ), the primates were re-challenged (see Table 3) at week $60 \mathrm{PI}$ as indicated by the double arrow. Each point represents the optical density value (mean \pm SD) at $492 \mathrm{~nm}$ of sera from five infected monkeys, as determined by ELISA. Individual humoral responses are also shown for the macaques N13 (that developed fulminating disease) and 015 (uninfected control). Broken line indicates the positive cut-off $(0.10 \pm 0.073)$ as determined by the mean of negative controls (representing pre-infection values of seven macaque sera) \pm 2 SD.

This type of immune response capable of creating a protective granuloma (Murray, 2001) is firmly linked to the IL-12-dependent IFN- $\gamma$ production pathway (Lammas et al., 2002).

Whilst the generation of anti-Leishmania antibodies apparently plays no role in resistance to leishmaniasis, a strong cell-mediated immune response is essential. The protective immune response in mice against $L$. major is dependent on the ability to mount an IL-12-driven T-helper cytokine type 1 (Th1) response, which activates infected macrophages by production of IFN- $\gamma$ for the killing of intracellular parasites (Alexander and Bryson, 2005). Vaccine efficacy against Leishmania is also thought to be largely associated with the emergence of a specific Th1 response (Coler and Reed, 2005). In humans, whilst there is a good correlation between Th1 responses and resistance to $\mathrm{CL}$, no association between increased IL- 4 and kala-azar has been identified (Kharazmi et al., 1999). However, a direct correlation between production of IL-10 and active disease is reported in patients with VL (Ghalib et al., 1993). One could speculate that the exacerbation of disease observed in macaque $\mathrm{N} 13$ could be due to the suppressor activity of $\mathrm{IL}-10$-secreting $\mathrm{CD}^{+} \mathrm{CD}_{25} 5^{+}$regulatory T-cells (Belkaid et al., 2002).

In the present study, $L$. infantum-specific antibodies in serum were detected by ELISA in all infected animals; levels of IgG antibodies rose during active infection and then declined during treatment, but they increased after rechallenge infection. Positive DTH responses were seen in most of the resistant monkeys with self-limiting disease when skin tested with leishmanin at 21 weeks after infection, whereas the control uninfected monkey and one monkey (N13) with progressive illness did not respond. Skin test reactivity following natural exposure is also thought to
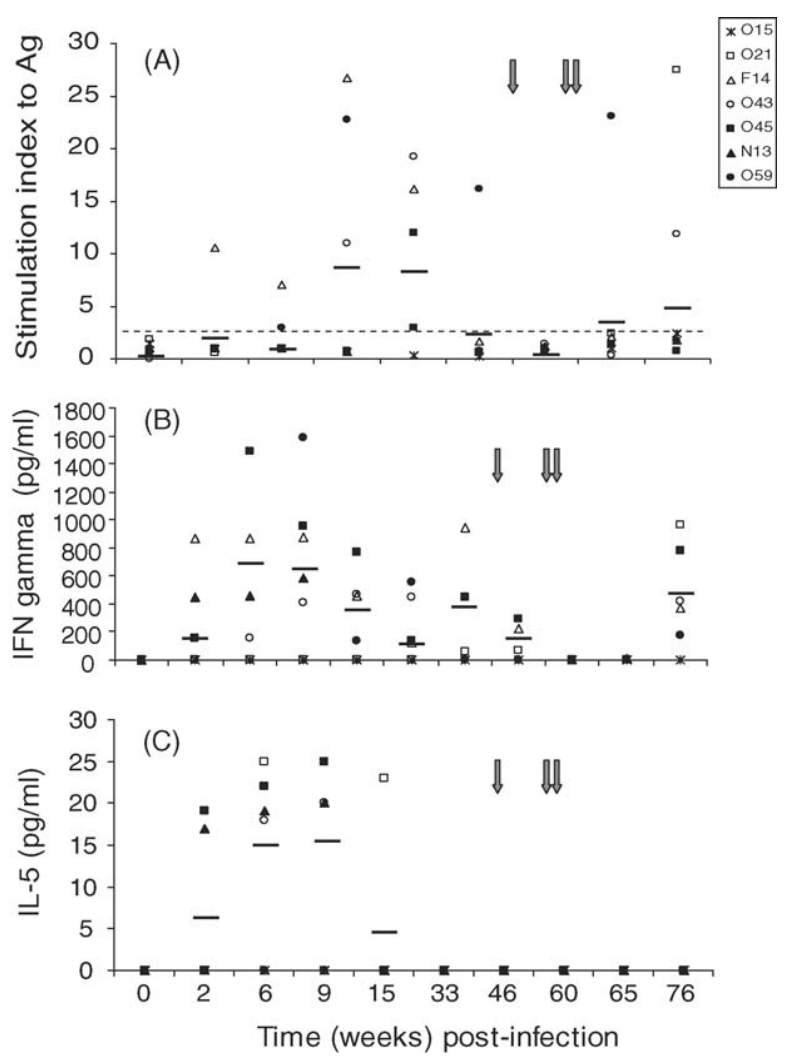

Figure 4 (A) Parasite-specific recall proliferative response and $(B, C)$ cytokine (IFN- $\gamma$ and IL-5) responses of T-lymphocytes from rhesus macaques prior to and after experimental infection with Leishmania infantum. After recovery from the primary infection (arrow indicates the start of antimonial therapy at week 37 post infection $(\mathrm{PI})$ ), the primates were subsequently re-challenged at week $60 \mathrm{PI}$ (double arrow). Suspensions of purified peripheral blood leukocytes were re-stimulated in vitro in the presence of soluble antigens (Ags) prepared from $L$. infantum. (A) Cell proliferation was assessed by measuring thymidine incorporation. Results are expressed as the stimulation index (SI; mean counts per minute of stimulated cultures/mean counts per minute of unstimulated cultures). Broken line indicates the positive cut-off value $(S I \geq 3)$. Determinations were made at minimum in triplicate. $(B, C)$ Cell culture medium samples collected after $72 \mathrm{~h}$ of stimulation were used for quantitative determinations of cytokine secretion in the supernatants by ELISA. Bars depict the mean SI, IFN- $\gamma$ and IL- 5 values of infected monkeys at different time points. Individual cellular immune responses are also indicated for the uninfected (control) monkey (015).

reflecting resistance to infection, with genetic epidemiology studies (Shaw et al., 1995) demonstrating a strong genetic component for mounting a skin test response to leishmanial antigen.

We have also used conventional in vitro assays of cellular immunity to understand some of the mechanisms that underlie resistance to $L$. infantum. The cytokines of interest were primarily selected because of their importance as markers for Th1 or Th2 immune responses or inflammatory responses. The anti-human IL-5 mAb evaluated in this study has been used previously for ELISA detection of 
macaque IL-5 (Mäkitalo et al., 2002). In our experiments, the antigen-stimulated cells produced IL-5 at low or undetectable levels, whereas production was slightly enhanced by PHA stimulation. This, and the finding (Mäkitalo et al., 2002) of the poor cross-reactivity displayed by the mAbs to cynomolgus and rhesus macaque IL-5, highlight the need for a thorough investigation of mAb cross-reactivity. Our results confirmed some studies on the basic immunological parameter in M. mulatta (Pahar et al., 2003), showing that the magnitude and kinetics of lymphocyte proliferation in response to antigen is not directly related to effector or T-cell functions such as secreted IFN- $\gamma$.

Despite the fact that clinical healing was not consistently linked with an abundance of antigen-specific IFN$\gamma$-producing cells, animals that recovered from previous infection acquired immunity to homologous re-infection. This was also the situation in vaccinated monkeys (CamposNeto et al., 2001; Kenney et al., 1999) in which neither pre-challenge in vitro antigen-specific recall T-cell proliferative and IFN- $\gamma$ responses nor positive DTH response were predictive of clinical protection. Taken together, this confirms the importance of quantitative assessment of lowfrequency, antigen-specific T-cells from rhesus macaques by ELISPOT and cytokine flow cytometry assays (Pahar et al., 2003), as well as using luminex technology (Giavedoni, 2005), to determine correlates of protection against leishmanial infection. Finally, the demonstration of a specific immunological response in $L$. infantum-infected macaques, such as described here, suggests that this primate model of human VL should be of practical importance since it shows several features underlying immunity that could be met by an effective vaccine.

\section{Conflicts of interest statement}

The authors have no conflicts of interest concerning the work reported in this paper.

\section{Acknowledgements}

We thank Dr Antonio da Mota Marinho and the staff in the Fiocruz Animal Care Facility for their assistance with daily care and procedures on the macaques. Financial support was received from Fiocruz; PRONEX $3 /$ CNPq (Brazil) and the Millennium Institute for Vaccine Development and Technology (CNPq-420067/2005-1).

\section{References}

Alexander, J., Bryson, K., 2005. T helper (h) 1/Th2 and Leishmania: paradox rather than paradigm. Immunol. Lett. 99, 1723.

Amaral, V.F., Ransatto, V.A.O., Conceição-Silva, F., Molinaro, E., Ferreira, V., Coutinho, S.G., McMahon-Pratt, D., Grimaldi Jr, G., 1996. Leishmania amazonensis: the Asian rhesus macaques (Macaca mulatta) as an experimental model for study of cutaneous leishmaniasis. Exp. Parasitol. 82, 34-44.

Amaral, V.F., Teva, A., Porrozzi, R., Silva, A.J., Pereira, M.S., Grimaldi Jr, G., 2001. Leishmania (Leishmania) major-infected rhesus macaques (Macaca mulatta) develop varying levels of resistance against homologous reinfections. Mem. Inst. Oswaldo Cruz 96, 795-804.

Andrade, M.C.R., Penedo, M.C.T., Ward, T., Silva, V.F., Bertolini, L.R., Roberts, J.A., Leite, J.P.G., Cabello, P.H., 2004a. Deter- mination of genetic status in a closed colony of rhesus monkeys (Macaca mulatta). Primates 45, 183-186.

Andrade, M.C.R., Ribeiro, C.T., Silva, V.F., Molinaro, E.M., Gonçalves, M.A.B., Pereira Marques, M.A., Cabello, P.H., Leite, J.P.G., 2004b. Biologic data of Macaca mulatta, Macaca fascicularis, and Saimiri sciureus used for research at the Fiocruz Primate Center. Mem. Inst. Oswaldo Cruz 99, 581589.

Belkaid, Y., Piccirillo, C.A., Mendez, S., Shevach, E.M., Sacks, D.L., 2002. $\mathrm{CD}^{+} \mathrm{CD}^{+} 5^{+}$regulatory $\mathrm{T}$ cells control Leishmania major persistence and immunity. Nature 420, 502-507.

Berman, J.D., Hanson, W.L., Chapman, W.L., Alving, C.R., LopezBerestein, G., 1986. Antileishmanial activity of liposomeencapsulated amphotericin $B$ in hamsters and monkeys. Antimicrob. Agents Chemother. 30, 847-851.

Binhazim, A.A., Shin, S.S., Chapman Jr, W.L., Olobo, J., 1993. Comparative susceptibility of African green monkeys (Cercopithecus aethiops) to experimental infection with Leishmania leishmania donovani and Leishmania leishmania infantum. Lab. Anim. Sci. 43, 37-47.

Broderson, J.R., Chapman Jr, W.L., Hanson, W.L., 1986. Experimental visceral leishmaniasis in the owl monkey. Vet. Pathol. 23, 293-302.

Campos-Neto, A., Porrozzi, R., Greeson, K., Coler, R.N., Webb, J.R., Seiky, Y.A., Reed, S.G., Grimaldi Jr, G., 2001. Protection against cutaneous leishmaniasis induced by recombinant antigens in murine and nonhuman primate models of the human disease. Infect. Immun. 69, 4103-4108.

Cartwright, G.D., Chung, H.L., Chang, A., 1948. Studies on the pancytopenia of kala-azar. Blood 3, 249-275.

Chapman Jr, W.L., Hanson, W.L., 1981. Visceral leishmaniasis in the squirrel monkey (Saimiri sciurea). J. Parasitol. 67, 740-741.

Chapman Jr, W.L., Hanson, W.L., Hendricks, L.D., 1981. Leishmania donovani in the owl monkey (Aotus trivirgatus). Trans. R. Soc. Trop. Med. Hyg. 75, 124-125.

Coler, R.N., Reed, S.G., 2005. Second-generation vaccines against leishmaniasis. Trends Parasitol. 21, 244-248.

Courtenay, O., Quinnell, R.J., Garcez, L.M., Shaw, J.J., Dye, C., 2002. Infectiousness in a cohort of Brazilian dogs: why culling fails to control visceral leishmaniasis in areas of high transmission. J. Infect. Dis. 186, 1314-1320.

Cupolillo, E., Grimaldi Jr, G., Momen, H., 1994. A general classification of a New World Leishmania using numerical zymotaxonomy. Am. J. Trop. Med. Hyg. 50, 296-311.

Davies, C.R., Kaye, P., Croft, S.L., Sundar, S., 2003. Leishmaniasis: new approaches to disease control. BMJ 326, 377-382.

Degrave, W., Fernandes, O., Campbell, D., Bozza, M., Lopes, U., 1994. Use of molecular probes and PCR for detection and typing of Leishmania - a mini-review. Mem. Inst. Oswaldo Cruz 89, 463-469.

Dennis, V.A., Chapman Jr, W.L., Hanson, W.L., Lujan, R., 1985. Leishmania donovani: clinical, haematological and hepatic changes in squirrel monkeys (Saimiri sciureus). J. Parasitol. 71, 576-582.

Dennis, V.A., Lujan, R., Chapman Jr, W.L., Hanson, W.L., 1986. Leishmania donovani: cellular and humoral immune responses after primary and challenge infections in squirrel monkeys, Saimiri sciureus. Exp. Parasitol. 61, 319-334.

Desjeux, P., 2001. The increase in risk factors for leishmaniasis worldwide. Trans. R. Soc. Trop. Med. Hyg. 95, 239-243.

Dube, A., Sharma, P., Srivastava, J.K., Misra, A., Naik, S., Katiyar, J.C., 1998. Vaccination of langur monkeys (Presbytis entellus) against Leishmania donovani with autoclaved $L$. major plus BCG. Parasitology 116, 219-221.

Dube, A., Srivastava, J.K., Sharma, P., Chaturvedi, A., Katiyar, J.C., Naik, S., 1999. Leishmania donovani: cellular and humoral immune responses in Indian langur monkeys Presbytis entellus. Acta Trop. 73, 37-48. 
Evans, T.G., Teixeira, M.J., Souza, A.Q., Pearson, R.D., 1995. Short report: extended follow-up of the natural history of persons infected with Leishmania chagasi. Am. J. Trop. Med. Hyg. 53, $360-361$.

Ghalib, H.W., Piuvezam, M.R., Skeiky, Y.A., Siddig, M., Hashim, F.A., el-Hassan, A.M., Russo, D.M., Reed, S.G., 1993. Interleukin 10 production correlates with pathology in human Leishmania donovani infections. J. Clin. Invest. 92, 324-329.

Giavedoni, L.D., 2005. Simultaneous detection of multiple cytokines and chemokines from nonhuman primates using luminex technology. J. Immunol. Methods 301, 89-101.

Gicheru, M.M., Olobo, J.O., Kariuki, T.M., Adhiambo, C., 1995. Visceral leishmaniasis in vervet monkeys: immunological responses during asymptomatic infections. Scand. J. Immunol. 41, 202-208.

Githure, J.I., Shatry, A.M., Tarara, R., Chulay, J.D., Suleman, M.A., Chunge, C.N., Else, J.G., 1986. The suitability of East African primates as animal models of visceral leishmaniasis. Trans. R. Soc. Trop. Med. Hyg. 80, 575-576.

Kenney, R.T., Sacks, D.L., Sypek, J.P., Vilela, L., Gam, A.A., EvansDavis, K., 1999. Protective immunity using recombinant human $\mathrm{IL}-12$ and alum as adjuvants in a primate model of cutaneous leishmaniasis. J. Immunol. 163, 4481-4488.

Kennedy, R.C., Shearer, M.H., Hildebrand, W., 1997. Nonhuman primate models to evaluate vaccine safety and immunogenicity. Vaccine 15, 903-908.

Kharazmi, A., Kemp, K., Ismail, A., Gasim, S., Gaafar, A., Kurtzhals, J.A., Hassan, A.M., Theander, T.G., Kemp, M., 1999. T-cell response in human leishmaniasis. Immunol. Lett. 65, 105-108.

Lammas, D.A., De Heer, E., Edgar, J.D., Novelli, V., BenSmith, A., Baretto, R., Drysdale, P., Binch, J., MacLennan, C., Kumararatne, D.S., Panchalingam, S., Ottenhoff, T.H.M., Casanova, J.L., Emile, J.F., 2002. Heterogeneity in the granulomatous response to mycobacterial infection in patients with defined genetic mutations in the interleukin 12-dependent interferon-gamma production pathway. Int. J. Exp. Pathol. 83, $1-20$.

Madindou, T.J., Hanson, W.L., Chapman Jr, W.L., 1985. Chemotherapy of visceral leishmaniasis (Leishmania donovani) in the squirrel monkey (Saimiri sciureus). Ann. Trop. Med. Parasitol. 79, 13-19.

Mäkitalo, B., Andersson, M., Areström, I., Karlén, K., Villinger, F., Ansari, A., Paulie, S., Throstensson, R., Ahlborg, N., 2002. ELISpot and ELISA analysis of spontaneous, mitogen-induced and antigen-specific cytokine production in cynomolgus and rhesus macaques. J. Immunol. Methods 270, 85-97.
Manson-Bahr, P.E.C., Wilson, V.L.C., 1976. Hepatic granulomas in monkeys infected with $L$. donovani. Trans. R. Soc. Trop. Med. Hyg. 70, 20.

Marsden, P.D., Cuba, C.C., Vexenat, A., Costa e Silva, M., Costa e Silva, A., Barreto, A.C., 1981. Experimental Leishmania chagasi infections in the marmoset Callithrix jacchus jacchus. Trans. R. Soc. Trop. Med. Hyg. 75, 314-315.

Misra, A., Dube, A., Srivastava, B., Sharma, P., Srivastava, J.K., Katiyar, J.C., Naik, S., 2001. Successful vaccination against Leishmania donovani infection in Indian langur using alum-precipitated autoclaved Leishmania major with BCG. Vaccine 19, 3485-3492.

Murray, H.W., 2001. Tissue granuloma structure-function in experimental visceral leishmaniasis. Int. J. Exp. Pathol. 82, 249-267.

Pahar, B., Li, J., Rourke, T., Miller, C.J., McChesney, M.B., 2003. Detection of antigen-specific $\mathrm{T}$ cell interferon $\gamma$ expression by ELISPOT and cytokine flow cytometry assays in rhesus macaques. J. Immunol. Methods 282, 103-115.

Schubach, A., Haddad, F., Oliveira-Neto, M.P., Degrave, W., Pirmez, C., Grimaldi Jr, G., Fernandes, O., 1998. Detection of Leishmania DNA by polymerase chain reaction in scars of treated human patients. J. Infect. Dis. 178, 911-914.

Sen Gupta, P.C., Chakravarty, N.K., Das Gupta, B., 1956. The liver in kala-azar. Ann. Trop. Med. Parasitol. 50, 252-259.

Shaw, M.A., Davies, C.R., Llanos-Cuentas, E.A., Collins, A., 1995. Human genetic susceptibility and infection with Leishmania peruviana. Am. J. Hum. Genet. 57, 1159-1169.

Shortt, H.E., 1923. The pathology of acute experimental kala-azar in monkeys. Indian J. Med. Res. 11, 186-195.

Stager, S., Smith, D.F., Kaye, P.M., 2000. Immunization with a recombinant stage-regulated surface protein from Leishmania donovani induces protection against visceral leishmaniasis. J. Immunol. 165, 7064-7071.

Teva, A., Porrozzi, R., Cupolillo, E., Pirmes, C., Oliveira-Neto, M.P., Grimaldi Jr, G., 2003. Leishmania (Viannia) braziliensis-induced chronic granulomatous cutaneous lesions affecting the nasal mucosa in the rhesus monkey (Macaca mulatta) model. Parasitology 127, 437-447.

Teva, A., Porrozzi, R., Cupolillo, E., Oliveira-Neto, M.P., Grimaldi Jr, G., 2005. Responses of Leishmania (Viannia) braziliensis cutaneous infection to $\mathrm{N}$-methylglucamine antimoniate in the rhesus monkey (Macaca mulatta) model. J. Parasitol. 91, 976978.

Volpini, A.C., Passos, V.M.A., Oliveira, G.C., Romanha, A.J., 2004. PCR-RFLP to identify Leishmania (Viannia) braziliensis and $L$. (Leishmania) amazonensis causing American cutaneous leishmaniasis. Acta Trop. 90, 31-37. 